\title{
Parâmetros genéticos de crescimento inicial e frutificação de Dipteryx alata Vogel em teste de progênie e espaçamento
}

\author{
Genetic parameters, initial growth and fruit production of Dipteryx alata \\ Vogel in progeny and spacing trial
}

\author{
Henrique Sverzut Freire de Andrade ${ }^{1}$, Gabriel de Resende Baroni ${ }^{1}$ (D), \\ Pedro Henrique Santin Brancalion ${ }^{1}$ (1), Mario Luiz Teixeira de Moraes ${ }^{2}$ (1), \\ Paulo Henrique Müller da Silva ${ }^{3}$ (1) \\ 1Escola Superior de Agricultura “Luiz de Queiroz" - ESALQ, Universidade de São Paulo - USP, Piracicaba, SP, Brasil \\ ${ }^{2}$ Faculdade de Engenharia de Ilha Solteira - FEIS, Universidade Estadual Paulista "Júlio de Mesquita Filho" - UNESP, Ilha \\ Solteira, SP, Brasil \\ ${ }^{3}$ Instituto de Pesquisas e Estudos Florestais - IPEF, Piracicaba, SP, Brasil
}

Como citar: Andrade, H. S. F., Baroni, G. R., Brancalion, P. H. S., Moraes, M. L. T., \& Silva, P. H. M. (2020). Parâmetros genéticos de crescimento inicial e frutificação de Dipteryx alata Vogel em teste de progênie e espaçamento. Scientia Forestalis, 48(126), e3074. https://doi.org/10.18671/scifor.v48n126.17

\begin{abstract}
Resumo
A espécie arbórea Dipteryx alata Vogel, conhecida como baru, ocorre no naturalmente no Cerrado. Sua madeira é valorizada em serrarias e os frutos possuem castanhas que são valorizadas para o consumo in natura e industrializado. Para reduzir a exploração em populações naturais são necessários estudos para o melhoramento genético e cultivo da espécie. Os objetivos foram estimar parâmetros genéticos, verificar o efeito de progênies e do espaçamento no crescimento e produção de frutos 30 meses após o plantio. O teste foi implantado no município de Rosário Oeste (MT) com 28 progênies de polinização aberta em oito espaçamentos (4,25 a 12,75 $\mathrm{m}^{2} \mathrm{pl}^{-1}$ ) em Nelder linear. Utilizou-se o delineamento casualizado em blocos, com três repetições e parcelas subdivididas em oito espaçamentos. Foram coletados dados de altura de plantas, diâmetro a altura do peito (DAP) e da presença de frutos (PF). Desses caracteres, foram estimados os parâmetros genéticos com o software Selegen utilizando o modelo 111 e o 54. Não houve efeito do espaçamento no crescimento e na presença de frutos até a idade avaliada. No entanto, houve efeito significativo de progênies nas duas variáveis de crescimento e na presença de frutos. Para altura, DAP e PF, respectivamente, os valores da: herdabilidade aditiva foram de 0,$38 ; 0,32 ; 0,25$; coeficiente de variação genética foram de 11,88\%; 11,53\%; 11,26\%; coeficiente de variação experimental foram de $26,30 \% ; 23,79 \% ; 21,93 \%$; coeficiente de variação relativa foram de 0,54 ; 0,48; 0,43; a média foi de 3,05m; 3,43cm; 10,41\%. Apesar de não ter ocorrido efeito do espaçamento, espera-se que o mesmo ocorra em idades posteriores. Houve variabilidade genética entre as progênies testadas e forte controle genético dos caracteres, assim há potencial para continuidade do programa de melhoramento da espécie.
\end{abstract}

Palavras-chave: Baru; Cerrado; Melhoramento genético; Nelder linear; Silvicultura de espécies nativas.

\begin{abstract}
The tree species Dipteryx alata Vogel, known as baru, occurs naturally in the Cerrado. Its wood is valued at sawmills and the fruits have nuts that are valued for consumption in natura and industrialized. To reduce exploitation in natural populations studies are needed for genetic improvement and cultivation of the species. The objectives were to estimate genetic parameters, verify the effect of progenies and spacing on growth and fruit yield 30 months after planting. The test was implanted in the municipality of
\end{abstract}

Fonte de financiamento: Parque Sesc Serra Azul, Coordenação de Aperfeiçoamento de Pessoal de Nível Superior - CAPES, Fundação de Amparo à Pesquisa do Estado de São Paulo - FAPESP.

Conflito de interesse: Nada a declarar.

Autor correspondente: gabrielbaroni92@gmail.com

Recebido: 10 setembro 2018.

Aceito: 22 julho 2019

Editor: Francides Gomes da Silva Júnior.

(c) (†) Este é um artigo publicado em acesso aberto (Open Access) sob a licença Creative Commons Attribution, que permite uso, distribuição e reprodução cc. em qualquer meio, sem restrições desde que o trabalho original seja corretamente citado. 
Rosário Oeste, MT with 28 progenies of open pollination in eight spacings (4.25 to $\left.12.75 \mathrm{~m}^{2} \mathrm{pl}^{-1}\right)$ in linear Nelder. The design was in a randomized block with three replications and the plots subdivided into eight spacings. Plant height, diameter at breast height (DAP) and fruit presence (PF) data were collected. Of these traits, the genetic parameters were estimated with the Selegen software using model 111 and 54. There was no effect of the spacing on the growth and presence of fruits until the evaluated age. However, there was a significant effect of progenies on the two growth traits and on the presence of fruits. For height, DAP and PF, respectively, the values of: additive heritability were $0.38 ; 0.32 ; 0.25$; coefficient of genetic variation were $11.88 \% ; 11.53 \% ; 11.26 \%$; coefficient of variation were of $26.30 \% ; 23.79 \% ; 21.93 \%$; coefficient of variation were $0.54 ; 0.48 ; 0.43$; the mean was $3.05 \mathrm{~m} ; 3.43 \mathrm{~cm} ; 10.41 \%$. Although no spacing effect occurs, it is expected that there will be effect at later ages. There was genetic variability between the tested progenies and strong genetic control of the characters, so there is potential for continuity of the breeding program of the species.

Keywords: Baru; Cerrado; Genetic improvement; Linear Nelder; Silviculture of native species.

\section{INTRODUÇÃO}

O baru ou cumbaru (Dipteryx alata Vogel, Fabaceae) é uma espécie arbórea nativa brasileira que ocorre no Cerrado e na sua transição com a Mata Atlântica. Essa espécie tem potencial econômico devido a sua madeira densa e resistente ao apodrecimento, adequada para a fabricação de mourões e obras externas (Lorenzi, 2014), também devido à sua castanha, que é apreciada pela população local, pode ser consumida in natura ou em produtos industrializados (Lorenzi et al., 2006; Lorenzi, 2014). Apesar de produzir anualmente grande quantidade de frutos, há registros de que a árvore inicia sua frutificação apenas aos 6 anos após o plantio (Carvalho, 2003). A exploração da madeira e fruto tem sido baseada em populações naturais, havendo carência de conhecimento sobre o cultivo da espécie, necessário para suprir uma crescente demanda do mercado.

Para adequar essa espécie, ainda não domesticada, ao sistema de cultivo florestal, é necessário o desenvolvimento de técnicas silviculturais relacionadas ao manejo e melhoramento genético. Além disso, são exigidos nas cadeias produtivas atuais alta qualidade e baixo custo de produção, porém trata-se de uma combinação difícil de se encontrar naturalmente nas espécies florestais. Com isso, há a necessidade do desenvolvimento de trabalhos visando maximizar o potencial de produção em sistemas de cultivo, indo além do extrativismo (Clement, 2001).

Quantificar a variabilidade genética é crucial para orientar os programas de melhoramento de espécies nativas. A variabilidade garante a adaptação frente às adversidades ambientais e biológicas que surgem ao longo do ciclo de cultivo das espécies florestais (Ribeiro \& Rodrigues, 2006; Botezelli et al., 2000).

Além da seleção de genótipos mais produtivos, é necessário conhecer os tratamentos silviculturais que favorecem a produção desses genótipos. Dentre esses, o espaçamento influencia no crescimento e produtividade das florestas, sendo que sua escolha depende do produto a ser gerado, características do sítio, operações silviculturais e custos de plantio (Stape \& Binkley, 2010; Lima et al., 2013; Hakamada et al., 2017).

Espaçamentos reduzidos favorecem a desrama natural e controle de mato-competição (Macedo et al., 2005; Caron et al., 2012). Quando inadequado, o adensamento excessivo reduz o crescimento e causa perdas de produtividade por competição ou estresse nutricional e hídrico (Moraes et al., 2013). Espaçamentos amplos favorecem o crescimento em diâmetro, porém exigem mais intervenções para o controle da mato-competição (Macedo et al., 2005; Caron et al., 2012) e desrama, quando se almeja madeira de maior qualidade para serraria (Paiva \& Leite, 2016).

O espaçamento influencia também na frutificação das árvores (Florence, 2004; Muniz, 2008; Wendling et al., 2014). Uma vez que o espaçamento influencia no tamanho da copa, na exposição à radiação solar e na atividade de polinizadores (Florence, 2004).

Para que o baru seja adotado em sistemas produtivos é preciso conhecer os parâmetros genéticos de populações para melhoramento e os efeitos de espaçamento no seu cultivo. Por meio dos parâmetros genéticos é possível mensurar a variabilidade e controle genético dos 
caracteres de interesse visando apoiar futuros programas de melhoramento. Já com o estudo do espaçamento é possível determinar a influência deste nos genótipos e caracteres estudados.

Os objetivos foram avaliar a influência do espaçamento e estimar os parâmetros genéticos de caracteres de crescimento e da produção de frutos de baru, visando subsidiar o cultivo da espécie em sistemas de produção silvicultural.

\section{MATERIAL E MÉTODOS}

\section{Coleta de sementes e produção de mudas}

Foram coletadas sementes de polinização aberta de 28 árvores matrizes de baru localizadas no município de Rosário Oeste (MT). A coleta foi realizada em julho de 2015 em árvores isoladas em pastagens e remanescentes de cerradão, sendo que foi considerada a distância mínima de 100 metros entre as árvores matrizes (Figura 1).

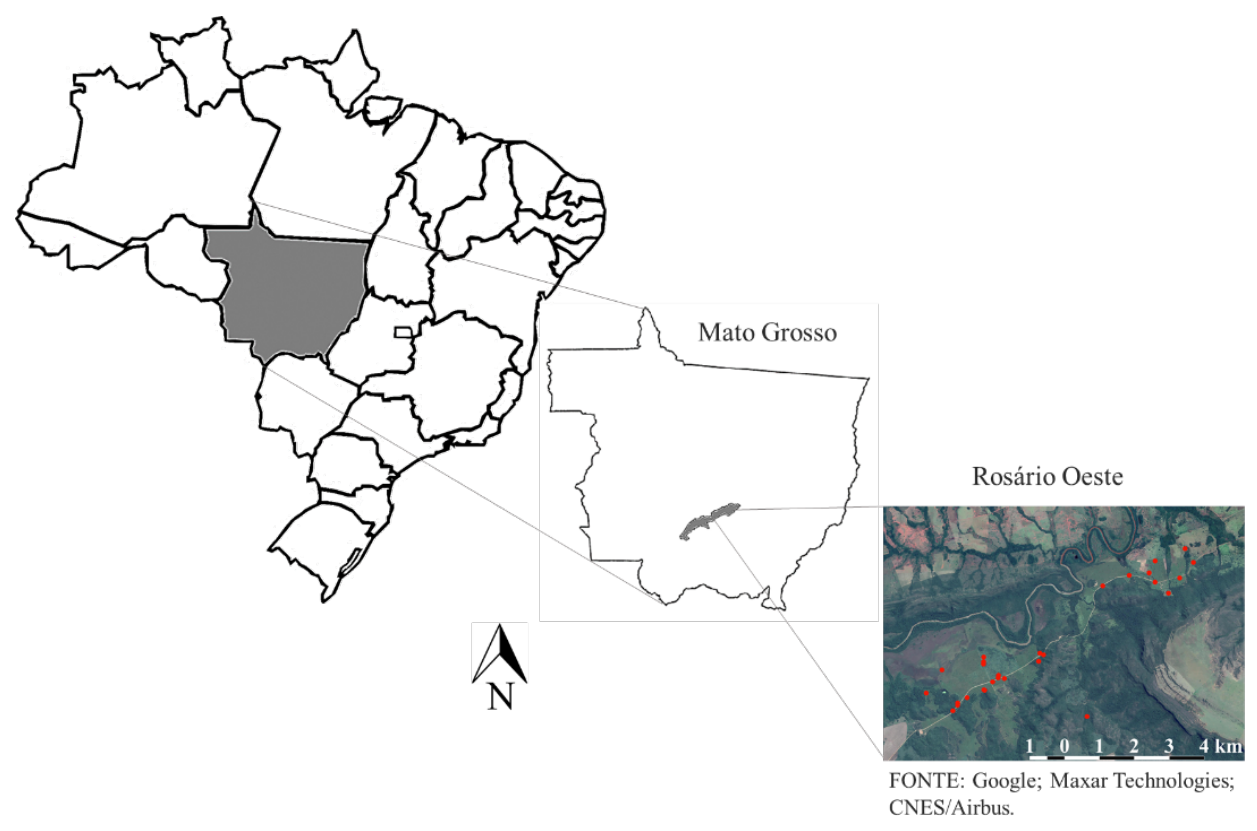

Figura 1 - Localização e distribuição espacial das árvores matrizes

Foram coletados no mínimo 30 frutos por árvore matriz. Esses frutos foram colocados em lonas estendidas na sombra durante 15 dias. Após esse período, os frutos foram transferidos para o viveiro em Piracicaba, SP. Posteriormente, as sementes foram retiradas dos frutos com o auxílio de um facão e foram transferidas para a câmara fria do viveiro, onde permaneceram armazenadas por 2 meses.

As mudas foram produzidas em Piracicaba (SP) onde o clima é Cwa, segundo a classificação de Köppen (1936), sendo subtropical de inverno seco. Assim, após o período de armazenamento, as sementes foram colocadas em tubetes de $280 \mathrm{~cm}^{3}$ preenchidos com substrato a base de proporções iguais de casca de arroz carbonizada, fibra de coco e vermiculita média. A fertirrigação foi realizada uma vez a cada 15 dias, sendo aplicada por meio de irrigação automática com a lâmina de água de $12 \mathrm{~mm}$. A solução de fertirrigação foi composta de $450 \mathrm{~g}$ nitrato de cálcio; $300 \mathrm{~g}$ de nitrato de amônio; $250 \mathrm{~g}$ de fosfato de monoamônio (MAP); $300 \mathrm{~g}$ de nitrato de potássio; $250 \mathrm{~g}$ de sulfato de magnésio; $250 \mathrm{~g}$ de sulfato de amônio $2,5 \mathrm{~g}$ de ferro tenso; $0,85 \mathrm{~g}$ de sulfato de manganês; $0,75 \mathrm{~g}$ de ácido bórico; $0,32 \mathrm{~g}$ de sulfato de zinco; 0,1 de sulfato de cobre e 0,005 g de molibdato de sódio. Todos esses sais foram diluídos em 1.000 litros de água. Recebendo esses tratos culturais, as mudas 
permaneceram no viveiro durante 12 meses e posteriormente foram transferidas para Rosário Oeste (MT), onde ocorreu a implantação do teste de progênie.

\section{Teste de progênie e espaçamento}

O teste foi implantado com 28 progênies de polinização aberta de baru no município de Rosário Oeste - MT, no bioma Cerrado. Nesta região, o clima é tropical com estação seca no inverno - Aw, segundo a classificação de Köppen (1936). As progênies foram obtidas de matrizes presentes na mesma propriedade e em áreas ao entorno do local onde o teste foi implantado.

O experimento foi implantado em Nelder Linear, testando 28 progênies e oito espaçamentos que variaram de 4,25 a 12,75 $\mathrm{m}^{2}$ por planta, sendo que o espaçamento entre linhas de plantio foi de 3 metros, enquanto entre plantas variou de 1,5 a 4,5 metros (Figura 2). O experimento foi organizado em parcelas subdivididas e o delineamento experimental casualizados em blocos com três repetições, sendo que cada linha foi formada por oito plantas da mesma progênie (parcela), sendo subdividida por oito espaçamentos (subparcela). A bordadura foi composta por uma linha de plantio ao redor de todo o limite do experimento.

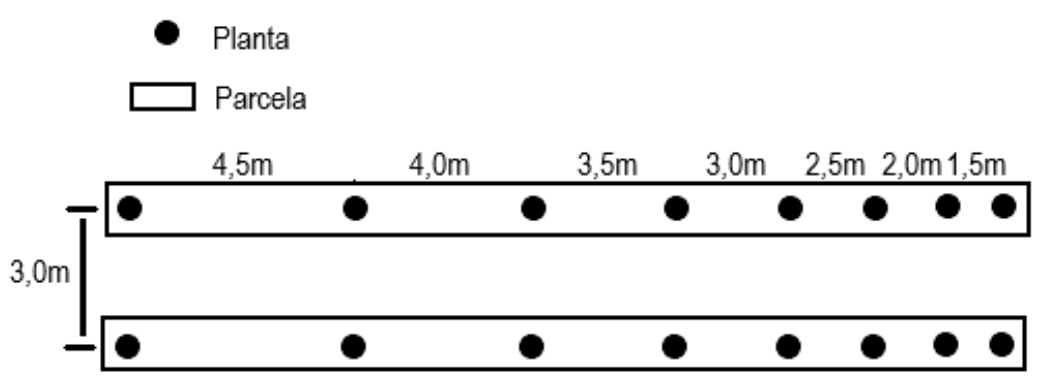

Figura 2 - Detalhamento da parcela experimental em Nelder Linear, composta por oito plantas de mesma progênie

O preparo da área de plantio, anteriormente ocupada por pastagem, foi realizado por meio de gradagem em área total. Posteriormente, as covas foram preparadas com $40 \mathrm{~cm}$ de profundidade e $15 \mathrm{~cm}$ de diâmetro. O plantio foi realizado em outubro de 2015 em uma área anteriormente ocupada por pastagem. Após sete dias foi realizada a adubação de base em covetas laterais, assim aplicou-se $100 \mathrm{~g}$ de NPK 5 - 25 - 15 por planta. Aos 15 meses após o plantio, foi realizada a adubação de cobertura com 150 g de NPK 20 - 5 - 15 por planta, juntamente com $50 \mathrm{~g}$ de formulação de micronutrientes. Quando necessário, foi realizada capina química com glifosato na linha de plantio. O controle de formigas cortadeiras foi intensivo até 30 meses após o plantio, por meio da distribuição de iscas formicidas pela área experimental e entorno.

\section{Coleta e análises de dados}

As avaliações foram realizadas 30 meses após o plantio. Foi mensurada a altura, o diâmetro à altura do peito (DAP, cm) e verificada a presença de frutos (PF, 1 ou 0).

Para verificar a diferença entre progênies foi realizado o Teste da Razão da Verossimilhança (LRT) com a diferença dos valores da deviance do modelo completo e com o modelo sem o efeito de progênies, sendo que os valores de deviance foram fornecidos pelo software Selegen (Resende \& Duarte, 2007). Em seguida, os parâmetros genéticos foram estimados pelo método REML/BLUP (Máxima Verossimilhança Restrita / Melhor Predição Linear Não Viesado) também pelo software SELEGEN (Resende \& Duarte, 2007). Para avaliar o efeito de interação genótipo por espaçamento $\left(C_{\text {int }}^{2}\right)$ e correlação genotípica entre o desempenho nos vários espaçamentos $\left(R g_{\text {esp }}\right.$ ) foi utilizado o modelo composto pelos efeitos: repetição; genótipo; interação genótipo por espaçamento (modelo 54). O efeito de repetição 
foi assumido como fixo e os demais como aleatórios. Posteriormente, foi calculada a média de cada caráter por espaçamento.

O modelo 111 foi utilizado para estimar os parâmetros: herdabilidade aditiva individual $\left(h_{a}^{2}\right)$; herdabilidade da média de progênies $\left(h_{m p}^{2}\right)$; acurácia da seleção de progênies $(A c)$; coeficiente de variação genotípica $\left(C V_{g} \%\right)$; coeficiente de variação residual ( $\left.C V_{e} \%\right)$; coeficiente de variação relativa $\left(C_{r}\right)$. Nesse modelo o efeito de repetição é considerado fixo enquanto os efeitos genéticos e resíduos foram considerados como aleatórios. Para utilizar esse modelo foi considerado que as plantas na linha do mesmo espaçamento eram do mesmo bloco, assim como havia 24 linhas foram considerados 24 repetições. Esse modelo é utilizado para espécies com sistema misto de reprodução, tal qual o baru. Assim, para o baru, o valor da taxa de autofecundação $(s)$ empregada no presente estudo foi de 0,55 (Tambarussi et al., 2017).

\section{RESULTADOS E DISCUSSÃO}

As árvores de baru apresentaram altura média de 3,0 m; DAP médio de 3,4 cm; 10,4\% dos indivíduos produziram frutos. A altura média no presente estudo foi superior a outros testes de progênies de baru tal como: Canuto et al. (2015) que apresentaram altura média de 1,5 m aos 21 meses e por Gomes (2011) que relatou altura média de 1,4 m aos 36 meses. Canuto et al. (2015) não mencionaram a realização de adubação nem de controle de plantas daninhas, enquanto Gomes (2011) relatou que o baixo crescimento em altura é decorrente da falta de adubação e outros tratos culturais.

O DAP médio do presente teste $(3,4 \mathrm{~cm})$ foi menor ao relatado por Siqueira et al. (1993) que apresentaram média de 4,45 cm variando de $5,88 \mathrm{~cm}$ a 2,07 cm entre as 5 procedências de baru avaliadas. Enquanto que no trabalho de Gomes (2011) o DAP médio do teste de progênies e procedências de baru foi de $5,11 \mathrm{~cm}$. Entretanto, cabe destacar que esses dois trabalhos foram avaliações aos 60 meses, ou seja, duas vezes a idade do presente teste. Ainda, cabe destacar que no primeiro o autor confirma que foi realizada a fertilização, já no segundo os autores não relataram a realização da fertilização mineral na metodologia do trabalho.

A maior altura média e o alto valor de DAP médio do presente estudo em comparação aos anteriormente citados pode ser devido aos tratos silviculturais empregados. Os tratos silviculturais beneficiaram o crescimento das plantas por causa do efeito positivo da adubação e do controle de plantas daninhas. Além disso, ressalta-se que nas espécies florestais existe influência do nível silvicultural não apenas no crescimento, mas também nos parâmetros genéticos obtidos nos testes de progênies (Silva et al., 2018).

O DAP médio do baru equivaleu a aproximadamente a metade do observado em Eucalyptus urophylla, que é a principal espécie utilizada para produção de madeira no cerrado. O valor médio de DAP do $E$. urophylla foi de $7,8 \mathrm{~cm}$ e $8,8 \mathrm{~cm}$, em dois testes de progênies aos 24 meses no cerrado (Pupin et al., 2015), porém sabe-se que E. urophylla vem sendo trabalhado ao longo das últimas décadas no Brasil visando aumentar a produtividade (Gonçalves et al., 2013). No entanto, a madeira de baru é usada para fins nobres e pode atingir valores de mercado superiores ao das espécies de eucalipto, além de gerar renda com a produção de castanha.

A produção de frutos é um caráter de avaliação inédita para o baru, por isso médias e parâmetros genéticos não foram encontrados na literatura para compará-los com os estimados no presente estudo. Deste caráter, cabe destacar a precocidade da frutificação das árvores que apresentaram frutos aos 30 meses, pois não há relatos de frutificação nessa idade, sendo que Carvalho (2003), Sano \& Fonseca (2003) relataram que a frutificação tem início a partir do sexto ano. Assim, a seleção de indivíduos com produção precoce de frutos pode ser realizada visando antecipar a produção de castanha para os potenciais produtores.

Os valores do coeficiente de determinação dos efeitos da interação genótipo versus espaçamento $\left(C_{\text {int }}^{2}\right)$ foram muito baixos $(0,01)$ para os três caracteres, enquanto os valores de correlação genotípica entre o desempenho nos vários espaçamentos ( $\left.R g_{\text {ecp }}\right)$ foram muito altos 
$(0,97)$ (Tabela 1). Os valores do primeiro parâmetro foram evidências de que o espaçamento não beneficiou nem prejudicou o crescimento e a frutificação das progênies. Enquanto os valores do segundo, demonstraram que as progênies possuem praticamente o mesmo desempenho em crescimento e frutificação entre os espaçamentos. Portanto, esses resultados são evidências de que o espaçamento não influenciou na expressão dos caracteres (Tabela 2). Isso ocorreu porque aos 30 meses ainda não havia competição entre indivíduos da população, mesmo nos espaçamentos mais adensados, de forma a não afetar os caracteres avaliados. No entanto, as avaliações em idades posteriores deverão apresentar diferença entre espaçamentos. A diferença acontecerá primeiramente nos espaçamentos reduzidos, pois com o crescimento as árvores iniciarão a competição por espaço e recursos. Quando as copas se tocarem as árvores competirão por luminosidade, enquanto as raízes, por água e nutrientes.

Binkley et al. (2017) avaliaram diversos clones de Eucalyptus spp. em 27 locais e relataram que o porte individual de todos os clones reduziu, 6 anos após o plantio, a medida em que se reduz da área útil da planta. Além disso, Moraes et al. (2013) estudando progênies de Jacaranda cuspidifolia, no delineamento sistemático em Nelder circular, verificaram que os valores médios de altura e DAP foram inversamente proporcionais à área útil das plantas.

Tabela 1: Parâmetros genéticos e teste LRT (Teste da Razão da Verossimilhança) nos caracteres altura, DAP (diâmetro à altura do peito) e PF (presença de frutos) em progênies de Dipteryx alata aos 30 meses em Rosário Oeste,MT

\begin{tabular}{ccccc}
\hline Modelo & Parâmetro Genético & Altura & DAP & PF \\
54 & $C_{\text {int }}^{2}$ & 0,01 & 0,01 & 0,01 \\
$R g_{e s p}$ & 0,97 & 0,97 & 0,97 \\
& LRT (qui-quadrado) & 109,31 * & 69,17 * & 60,57 * \\
& $h_{a}^{2}$ & 0,38 & 0,32 & 0,25 \\
$A c$ & 0,93 & 0,92 & 0,90 \\
$C V_{g}(\%)$ & 11,88 & 11,53 & 11,26 \\
$C V_{e}(\%)$ & 21,93 & 23,79 & 26,30 \\
$C V_{r}$ & 0,54 & 0,48 & 0,43 \\
& Média & $3,05 \mathrm{~m}$ & $3,43 \mathrm{~cm}$ & $10,41 \%$ \\
\hline
\end{tabular}

Legenda: $C_{\text {int }}^{2}$ :interação genótipo x ambiente (espaçamento); $R g_{\text {esp }}$ correlação genotípica entre o desempenho nos espaçamentos; $h_{a}^{2}$ : herdabilidade aditiva individual; $h_{m p}^{2}$ : herdabilidade da média de progênies; $A c:$ acurácia; $C V_{g}$ : coeficiente de variação genética; $C V_{e}$ : coeficiente de variação experimental.

Tabela 2: Médias de altura, DAP (diâmetro à altura do peito) e PF (presença de frutos) aos 30 meses por espaçamento em progênies de Dipteryx alata em Rosário Oeste,MT

\begin{tabular}{cccc}
\hline Espaçamento $\left.\mathbf{( m}^{\mathbf{2}}\right)$ & Altura $(\mathbf{m})$ & DAP $(\mathbf{c m})$ & PF (\%) \\
13,50 & 2,94 & 3,28 & 11,90 \\
12,75 & 3,07 & 3,47 & 7,14 \\
11,25 & 3,17 & 3,50 & 7,14 \\
9,75 & 3,06 & 3,48 & 10,71 \\
8,25 & 3,10 & 3,58 & 14,29 \\
6,75 & 3,08 & 3,46 & 10,71 \\
5,25 & 3,05 & 3,36 & 8,33 \\
4,50 & 2,95 & 3,33 & 13,10 \\
Média Geral & 3,05 & 3,43 & 10,41 \\
\hline
\end{tabular}


Os caracteres estudados (altura, DAP e PF) apresentaram diferença significativa entre progênies (Tabela 1) de acordo com o LRT. A população apresentou valores altos de herdabilidade aditiva ( $h_{a}^{2}: 0,25$ a 0,38), também valores altos para herdabilidade da média de progênies $\left(h_{m p}^{2}: 0,81\right.$ a 0,87). Já os valores de acurácia da seleção de genótipos foram considerados muito altos, pois foram maiores que 0,90 (Resende \& Duarte, 2007). Sabe-se que em espécies com sistema misto de reprodução o s pode variar de $5 \%$ a 95\% (Vencovsky et al., 2001), assim é essencial que a estimativa da variância aditiva passe pela correção considerando o s. Se o baru fosse considerado como espécie alógama, o que muitos pesquisadores realizam para espécies com sistema misto de reprodução (Tambarussi et al., 2018), a estimativa da variância aditiva seria calculada conforme $V_{g}=\frac{1}{4}\left(V_{a}\right)$. Entretanto, tendo o $s$ é possível realizar a correção considerando que $V_{g}=\frac{(1+s)^{2}}{4} \cdot\left(V_{a}\right)$, sendo $V_{g}$ a variância genotípica e $V_{a}$ é a variância aditiva. Segundo Tambarussi et al. (2018) quando essa correção não é realizada a herdabilidade pode ser superestimada em até $32 \%$ dependendo da espécie e do caráter, assim enviesando o cálculo do ganho genético no programa de melhoramento.

A $h_{a}^{2}$ para o caráter altura $(0,38)$ foi maior que valores relatados por Rocha et al. (2009), Gomes (2011) e Canuto et al. (2015) que apresentaram, respectivamente, 0,26; 0,22; e 0,25 para altura de baru, sendo que os dois primeiros foram estudos em populações aos 36 meses e o último estudo foi com 21 meses após o plantio. A $h_{a}^{2}$ do caráter DAP $(0,32)$ também foi similar ao relatado por Gomes (2011) que obteve 0,34 em baru aos 60 meses e superior a Zaruma et al. (2015) que apresentaram 0,27 em baru aos 9 anos. No entanto, foi superior Pagliarini et al. (2016) que apresentaram 0,06 em baru aos 25 anos.

Os valores de $\mathrm{CV}_{\mathrm{g}}$ variaram de $11,3 \%$ a $11,9 \%$, considerando todos os caracteres. Esses valores foram considerados altos, pois foram superiores a 7\%, valor normalmente utilizado de base para comparação (Sebbenn et al., 1998). Tais valores foram similares a Rocha et al. (2009) que obtiveram 13,3\% e inferiores a Canuto et al. (2015) que apresentaram 26,9\%, ambos para altura em baru, sendo o primeiro estudo em população aos 3 anos e o último aos 21 meses após plantio. Ainda, os valores de $\mathrm{CV}_{\mathrm{g}} \%$ para DAP foram inferiores a Zaruma et al. (2015) que apresentaram valores variando de 17,1\% a 31,1\% em teste de progênies e procedências de baru aos 9 anos e similares a Pagliarini et al. (2016) que apresentaram 10,9\% em baru aos 25 anos.

De acordo com Pimentel-Gomes (2009) os valores de $\mathrm{CV}_{e}$ nos caracteres de crescimento foram considerados altos, pois estiveram entre $20 \%$ e $30 \%$. No entanto, esses valores de $\mathrm{CV}_{e}$ foram similares aos relatados por Moraes et al. (2013) que utilizaram delineamento sistemático circular de Nelder em teste de progênies de J. cuspidifolia e que apresentou valores de 25,8 e 31,7\% para altura aos 12 e 24 meses. Assim, considerou-se que o $\mathrm{CV}_{e}$ foi adequado aos testes de campo com outras espécies nativas e utilizando delineamento parecido.

$\mathrm{O}$ valores de $\mathrm{CV}_{r}$ para os caracteres altura, DAP e PF, foram respectivamente, de 0,54; 0,$48 ; 0,43$. De acordo com Resende \& Duarte (2007), para as 24 repetições consideradas, esses valores de $\mathrm{CV}_{r}$ foram considerados altos.

A existência de diferença entre progênies para os caracteres avaliados, os altos valores de $\mathrm{CV}_{g} \%, C V_{r}$ e $\mathrm{Ac}$ significam que houve variação genética entre indivíduos, entre progênies, confiabilidade e precisão de seleção (Resende \& Duarte, 2007; Pires et al., 2011). Além disso, os caracteres possuíram forte controle genético. Portanto, recomenda-se a seleção entre e dentro de progênies, pois a variação genética entre indivíduos e entre progênies devem ser preservadas. Assim, a seleção de indivíduos superiores proporcionará o ganho genético e o avanço da geração de melhoramento, enquanto que manter o máximo possível de progênies permitirá a conservação genética, pois essa população servirá de base para continuidade do programa de melhoramento (Pires et al., 2011) 


\section{CONCLUSÃO}

Não houve efeito do espaçamento em nenhum dos caracteres, mas houve efeito significativo das progênies no crescimento e frutificação aos 30 meses. Além disso, os valores dos parâmetros genéticos mostraram que existe forte controle genético e presença de variabilidade genética entre as progênies. Isso demonstrou que existe potencial positivo com a continuidade do programa de melhoramento genético do baru.

\section{AGRADECIMENTOS}

Agradecemos ao Parque Sesc Serra Azul, à Coordenação de Aperfeiçoamento de Pessoal de Nível Superior, ao Programa Cooperativo sobre Melhoramento Florestal (PCMF) do Instituto de Pesquisa e Estudos Florestais (IPEF) por todo fomento e apoio disponibilizados a realização desse trabalho.

\section{REFERÊNCIAS}

Binkley, D., Campoe, O. C., Alvares, C., Carneiro, R. L., Cegatta, Í., \& Stape, J. L. (2017). The interactions of climate, spacing and genetics on clonal Eucalyptus plantations across Brazil and Uruguay. Forest Ecology and Management, 405, 271-283. http://dx.doi.org/10.1016/j.foreco.2017.09.050.

Botezelli, L., Claudio, A., \& Malavasi, M. M. (2000). Características dos frutos e sementes de quatro procedências de Dipteryx alata Vogel (Baru). Revista Cerne, 6(1), 9-18.

Canuto, D. S. O., Zaruma, D. U. G., Moraes, M. A., Silva, A. M., Moraes, M. L. T., \& Freitas, M. L. M. (2015). Caracterização genética de um teste de progênies de Dipteryx alata Vog. proveniente de remanescente florestal da Estação Ecológica de Paulo de Faria, SP, Brasil. Hoehnea, 42(4), 641-648. http://dx.doi.org/10.1590/2236-8906-13/RAD/2015.

Caron, B. O., Lamego, F. P., Souza, V. Q., Costa, E. C., Eloy, E., Behling, A., \& Trevisan, R. (2012). Interceptação da radiação luminosa pelo dossel de espécies florestais e sua relação com o manejo das plantas daninhas. Ciência Rural, 42(1), 75-82. http://dx.doi.org/10.1590/S010384782012000100013.

Carvalho, P. E. R. (2003). Espécies arbóreas brasileiras (Vol. 1. 1039 p.). Brasília: EMBRAPA Informação Tecnológica; Colombo: EMBRAPA Florestas.

Clement, C. R. (2001). Melhoramento de espécies nativas. In L. L. Nass, A. C. C. Valois, I. S. Melo \& M. C. Valadares-Inglis (Eds.), Recursos genéticos \& Melhoramento - plantas (pp. 423-441). Rondonópolis: Fundação MT. Recuperado em 12 de abril de 2018, de HTTPS://www.inpa.gov.br/cpca/charles/pdf/spp-nativas.pdf

Florence, R. (2004). Ecology and silviculture of eucalypt forests. Collingwood: Csiro Publishing. http://dx.doi.org/10.1071/9780643100893.

Gomes, J. E. (2011). Variabilidade genética e correlações juvenil - adulto de baru (Dipteryx alata Vog.) no Município de Brasilândia - MG. Recuperado em 12 de abril de 2018, de http://repositorio.unesp.br/bitstream/handle/11449/106670/gomes_je_dr_botfca.pdf?sequence=1\& isAllowed=y

Gonçalves, J. L. M., Alvares, C. A., Higa, A. R., Silva, L. D., Alfenas, A. C., Stahl, J., Ferraz, S. F. B., Lima, W. P., Brancalion, P. H. S., Hubner, A., Bouillet, J. P. D., Laclau, J. P., Nouvellon, Y., \& Epron, D. (2013). Integrating genetic and silvicultural strategies to minimize abiotic and biotic constraints in Brazilian eucalypt plantations. Forest Ecology and Management, 301, 6-27. http://dx.doi.org/10.1016/j.foreco.2012.12.030.

Hakamada, R., Hubbard, R. M., Ferraz, S., Stape, J. L., \& Lemos, C. (2017). Biomass production and potential water stress increase with planting density in four highly productive clonal Eucalyptus genotypes. Southern Forests, 79(3), 251-257. http://dx.doi.org/10.2989/20702620.2016.1256041.

Köppen, W. (1936). Das geographische system der klimate. In W. Köppen \& R. Geiger (Eds.), Handbuch der Klimatologie (pp. 1-44). Berlin: Gebrüder Bornträger.

Lima, R., Inoue, M. T., Figueiredo Filho, A., Araujo, A. J., \& Machado, S. A. (2013). Efeito do espaçamento no desenvolvimento volumétrico de Pinus taeda L. Floresta e Ambiente, 20(2), 223-230. http://dx.doi.org/10.4322/floram.2013.001.

Lorenzi, H. J. (2014). Árvores brasileiras: manual de identificação e cultivo de plantas arbóreas nativas do Brasil. (Vol. 1, 6. ed.). São Paulo: Instituto Plantarum de Estudos da Flora. 
Lorenzi, H. J., Bacher, L., Lacerda, M., \& Sartori, S. (2006). Frutas brasileiras e exóticas cultivas: (de consumo in natura). São Paulo: Instituto Plantarum de Estudos da Flora.

Macedo, R. L. G., Gomes, J. E. G., Venturini, N., \& Salgado, B. G. (2005). Desenvolvimento inicial de Tectona grandis L.f. (teca) em diferentes espaçamentos no município de Paracatu, MG. Cerne, 11(1), 61-69.

Moraes, M. A., Moraes, S. M. B., Silva, E. C. B., Kubota, T. Y. K., Silva, A. M., Resende, M. D. V., \& Moraes, M. L. T. (2013). Variação genética em progênies de Jacaranda cuspidifolia Mart. utilizando o delineamento sistemático tipo "leque”. Scientia Forestalis, 41(98), 175-183.

Muniz, F. H. (2008). Padrões de floração e frutificação de árvores da Amazônia Maranhense. Acta Amazonica, 38(4), 617-626. http://dx.doi.org/10.1590/S0044-59672008000400004.

Pagliarini, M. K., Konrad, E. C. G., Silva, F. C., Silva, M. S. C., Moreira, J. P., Sato, A. S., Machado, J. A. R., Freitas, M. L. M., Aguiar, A. V., Moraes, M. L. T., \& Sebbenn, A. M. (2016). Variação genética em caracteres de crescimento em progênies de Dipteryx alata Vog. Scientia Forestalis, 44(112), 925-935. http://dx.doi.org/10.18671/scifor.v44n112.14

Paiva, H. N., \& Leite, H. G. (2016). Desbastes e desramas em povoamentos de Eucalyptus. In M. V. Schumacher \& M. Vieira. Silvicultura do Eucalipto no Brasil. Santa Maria: Editora UFSM.

Pimentel-Gomes, F. (2009). Curso de estatística experimental (15. ed.). Piracicaba: FEALQ.

Pires, I. E., Resende, M. D. V., Silva, R. L., \& Resende-Júnior, M. F. R. (2011). Genética florestal. Viçosa: Editora UFV.

Pupin, S., Santos, A. V. A., Zaruma, D. U. G., Miranda, A. C., Silva, P. H. M., Marino, C. L., Sebbenn, A. M., \& Moraes, M. L. T. (2015). Produtividade, estabilidade e adaptabilidade em progênies de polinização aberta de Eucalyptus urophylla S.T. Blake. Scientia Forestalis, 43(105), 127-134.

Resende, M. D. V., \& Duarte, J. B. (2007). Precisão e controle de qualidade em experimentos de avaliação de cultivares. Pesquisa Agropecuária Tropical, 37(3), 182-194.

Ribeiro, R. A., \& Rodrigues, F. M. (2006). Genética da conservação em espécies vegetais do cerrado. Revista de Ciências Médicas e Biológicas, Salvador, 5(3), 253-260.

Rocha, R. B., Rocha, M. G. B., Santana, R. C., \& Vieira, A. H. (2009). Estimação de parâmetros genéticos e seleção de procedências e famílias de Dipteryx alata Vogel (baru) utilizando metodologia de REML/BLUP e E(QM). Cerne, 15(3), 331-228.

Sano, S. M., \& Fonseca, C. E. L. (2003). Taxa de sobrevivência e frutificação de espécies nativas do cerrado. Planaltina: Embrapa Cerrados.

Sebbenn, A. M., Siqueira, A. C. M. F., Kageyama, P. Y., \& Machado, J. A. R. (1998). Parâmetros genéticos na conservação da cabreúva (Myroxylon peruiferum L.F. Allemão). Scientia Forestalis, 53, 31-38.

Silva, P. H. M., Brune, A., Pupin, S., Moraes, M. L. T., Sebbenn, A. M., \& Paula, R. C. (2018). Maintenance of genetic diversity in Eucalyptus urophylla S. T. Blake populations with restriction of the number of trees per family. Silvae Genetica, 67(1), 34-40. http://dx.doi.org/10.2478/sg-2018-0005.

Siqueira, A. C. M. D. F., Nogueira, J. C. B., \& Kageyama, P. Y. (1993). Conservação dos recursos genéticos ex situ do cumbaru (Dipteryx alata) Vog. - Leguminosae. Revista do Instituto Florestal, 5(2), 231-243.

Stape, J. L., \& Binkley, D. (2010). Insights from full-rotation Nelder spacing trials with Eucalyptus in São Paulo, Brazil. Southern Forests, 72(2), 91-98. http://dx.doi.org/10.2989/20702620.2010.507031.

Tambarussi, E. V., Pereira, F. B., Silva, P. H. M., Lee, D., \& Bush, D. (2018). Are tree breeders properly predicting genetic gain? A case study involving Corymbia species. Euphytica, 214(8), 150. http://dx.doi.org/10.1007/s10681-018-2229-9.

Tambarussi, E. V., Sebbenn, A. M., Alves-Pereira, A., Vencovsky, R., Cambuim, J., Da Silva, A., Moraes, M., \& Moraes, M. L. T. (2017). Dipteryx alata Vogel (Fabaceae), a neotropical tree with high levels of selfing: implications for conservation and breeding programs. Annals of Forest Research, 60(2), 243261. http://dx.doi.org/10.15287/afr.2017.842.

Vencovsky, R., Pereira, M. B., Crisostomo, J. R., \& Ferreira, M. A. J. F. (2001). Genética e melhoramento de populações mistas. In L. L. Nass, A. C. C. Valois, I. S. Melo \& M. C. Valadares-Inglis (Eds.), Recursos genéticos e melhoramento - plantas. Rondonópolis: Fundação MT.

Wendling, I., Trueman, S. J., \& Xavier, A. (2014). Maturation and related aspects in clonal forestry-Part I: Concepts, regulation and consequences of phase change. New Forests, 45(4), 449-471. http://dx.doi.org/10.1007/s11056-014-9421-0.

Zaruma, D. U. G., Canuto, D. S. O., Pupin, S., Cambuim, J., Silva, A. M., Mori, E. S., Sebbenn, A. M., \& Moraes, M. L. T. (2015). Variabilidade genética em procedências e progênies de Dipteryx alata vogel para fins de conservação genética e produção de sementes. Scientia Forestalis, 43(107), 609-615. 
Contribuição dos Autores: HSFA: conceituação, curadoria de dados, investigação, administração do projeto, visualização, escrita - primeira redação, escrita - revisão e edição; GRB: Análise formal, metodologia, escrita primeira redação, visualização, escrita - revisão e edição; MLTM: Análise formal, metodologia, supervisão, validação; PHSB: conceituação, obtenção de financiamento, administração do projeto, supervisão, validação, escrita - revisão e edição; PHMS: conceituação, obtenção de financiamento, investigação, metodologia, administração do projeto, supervisão, validação, visualização, escrita - revisão e edição. 